Pacific Journal of Mathematics

NONANALYTIC FUNCTIONAL CALCULI AND SPECTRAL 


\title{
NON-ANALYTIC FUNCTIONAL CALCULI AND SPECTRAL MAXIMAL SPACES
}

\author{
Philip C. Curtis, JR. And Michael M. Neumann \\ Dedicated to the memory of Henry A. Dye
}

\begin{abstract}
We are concerned with the algebraic representation of the spectral maximal spaces for certain classes of decomposable operators on Banach spaces. The main emphasis will be on those operators which admit a functional calculus on a suitable algebra of functions such as, for instance, differentiable functions, Lipschitz functions, or functions with absolutely convergent Fourier series. Using appropriate partitions of unity, we shall give a unified approach to various previous results in this area as well as to certain extensions and variants thereof. In the case of spectral operators, we shall obtain the optimal results without any assumption on the underlying Banach space or the Boolean algebra of projections corresponding to the spectral measure.
\end{abstract}

0. Introduction and motivation. If $E$ denotes the spectral measure associated with a bounded normal operator $T$ on a complex Hilbert space $X$, then the ranges of the spectral projections can be represented in the form

$$
E(F) X=\bigcap_{\lambda \in \mathbf{C} \backslash F}(T-\lambda) X \quad \text { for all closed } F \subseteq \mathbf{C} .
$$

This remarkable description of the spectral measure in purely algebraic terms was obtained independently by Putnam [19] and by Pták and Vrbová [17] after a slightly weaker result due to Johnson [9]. Apart from its intrinsic value, the algebraic representation of the spaces $E(F) X$ turned out to be particularly useful for the automatic continuity of linear transformations intertwining a given pair of normal operators [9], [21]. In order to handle the same kind of automatic continuity problem in the more general setting of generalized scalar operators [4], Vrbová [23] proved a similar representation theorem for the spectral maximal spaces $X_{T}(F)$ of a generalized scalar operator $T$ on a complex Banach space $X$. In this case, the best one can hope for is the algebraic representation

$$
X_{T}(F)=\bigcap_{\lambda \in \mathbf{C} \backslash F}(T-\lambda)^{p} X \quad \text { for all closed } F \subseteq \mathbf{C}
$$


with a suitable integer $p \geq 1$. With respect to this exponent, the result of Vrbová [23] was then improved by Foiaş and Vasilescu [8] and finally extended to the case of scalar systems of several commuting operators by Albrecht and Vasilescu [3]; see also [22]. A somewhat related representation theorem for certain spectral subspaces involving Banach modules over a locally compact abelian group $G$ was recently given by Johnson [10]. This result turned out to be useful in connection with the automatic continuity problem for homomorphisms of Banach $G$-modules.

In the present paper, we attempt to give a simple and unified approach to the algebraic representation of the spectral maximal spaces for certain decomposable operators [4], [22]. The basic idea is to combine the beautiful geometric argument from the classical paper of Johnson [9] with some elementary techniques on partitions of unity in spaces of differentiable functions. This approach avoids the somewhat complicated methods from previous papers, which have to invoke some general principles like the Baire category theorem and require specific results from the theory of generalized scalar operators. It turns out that our more elementary approach yields the best known results for generalized scalar operators and can be easily extended to other classes of operators which possess a (non-analytic) functional calculus on a suitable algebra of functions with partitions of unity. This includes, for instance, the case of Lipschitz functions or functions with absolutely convergent Fourier series, but we shall also give a counterexample for a certain non-quasianalytic class of functions. Moreover, in the case of spectral operators, we shall obtain the best possible results without any assumption on the underlying Banach space or the Boolean algebra of projections associated with the spectral measure.

The organization of this article is as follows. In $\S 1$, we discuss the algebraic representation of the spectral maximal spaces for some general classes of decomposable operators [4], [22]. In the case of certain multiplication and convolution operators, we are able to improve the weak kind of representation obtained in [13]. In $\S 2$, we collect some auxiliary results on partitions of unity for differentiable functions. Here, the main point is to control the norms of the derivatives for long products of differentiable functions; these estimates may be of some independent interest. In $\S 3$, we finally present our main results on non-analytic functional calculi and spectral maximal spaces. For simplicity, we restrict ourselves to the investigation of one single operator, but our methods can certainly be expanded to the case 
of finite systems of several commuting operators. For applications in automatic continuity theory, we refer to [9], [12], [13], [15], [21].

Most of the results of the present paper were obtained at the Workshop on Derivations and Radical Banach Algebras at Berkeley in July 1986. It is a pleasure to acknowledge the stimulating atmosphere of this conference; we are particularly grateful to Garth Dales for several valuable conversations.

1. Some reduction principles. Given $T \in \mathscr{L}(X)$, the continuous linear operators on some complex Banach space $X$, let Lat $(T)$ stand for the collection of all closed $T$-invariant linear subspaces of $X . T$ is called decomposable, if for every open covering $\{U, V\}$ of the complex plane $\mathbf{C}$, there exist $Y, Z \in \operatorname{Lat}(T)$ such that $Y+Z=X$ as well as $\sigma(T \mid Y) \subseteq U$ and $\sigma(T \mid Z) \subseteq V$, where $\sigma$ denotes the spectrum. We refer to the monographs [4] and [22] for the theory of decomposable operators. An important feature in this theory is the investigation of the spectral maximal spaces given by

$$
X_{T}(F)=\left\{x \in X: \sigma_{T}(x) \subseteq F\right\} \text { for all closed } F \subseteq \mathbf{C},
$$

where $\sigma_{T}(x) \subseteq \mathbf{C}$ denotes the local spectrum of the decomposable operator $T \in \mathscr{L}(X)$ at the point $x \in X$ in the sense of [4], [22]. Here, we are interested in representing the spaces $X_{T}(F)$ in terms of the ranges $(T-\lambda)^{p} X$ for suitable $p \in \mathbf{N}$ and $\lambda \in \mathbf{C}$. In the present section, this problem will be reduced to the much simpler case $F=\varnothing$ for some wide classes of decomposable operators; see also [8], [22] for the special case of generalized scalar operators and [1], [12], [13] for some related reduction principles of a similar algebraic flavor.

We shall consider the following subclasses of the decomposable operators. An operator $T \in \mathscr{L}(X)$ is said to be super-decomposable [13], if for every open covering $\{U, V\}$ of $\mathbf{C}$, there exists some $R \in \mathscr{L}(X)$ such that $R T=T R, \sigma(T \mid \overline{R(X)}) \subseteq U$, and $\sigma(T \mid \overline{(I-R)(X)}) \subseteq V$. More generally, $T \in \mathscr{L}(X)$ is called well-decomposable [1], if for every open covering $\{U, V\}$ of $\mathbf{C}$, there exist an $R \in \mathscr{L}(X)$, an integer $n \in \mathbf{N}$ and $Y, Z \in \operatorname{Lat}(T)$ such that $R(X) \subseteq Y,(I-R)(X) \subseteq$ $Z, \sigma(T \mid Y) \subseteq U, \sigma(T \mid Z) \subseteq V$, and finally $C(T, T)^{n} R=0$. The commutator $C(S, T): \mathscr{L}(X) \rightarrow \mathscr{L}(X)$, for an arbitrary pair of operators $S, T \in \mathscr{L}(X)$, is defined by

$$
C(S, T) A=S A-A T \text { for all } A \in \mathscr{L}(X) .
$$

It can be shown by examples that the exponent $n \in \mathbf{N}$ occurring in the last definition actually depends on the given covering and cannot 
be chosen uniformly in general. Well-decomposable operators and, in particular, super-decomposable operators are obviously decomposable and in fact strongly decomposable; for more information we refer to [1], [7], [13].

1.1. Proposition. Let $T \in \mathscr{L}(X)$ be super-decomposable such that

$$
\bigcap_{\lambda \in \mathbf{C}}(T-\lambda)^{p} X=\{0\}
$$

for some $p \in \mathbf{N}$. Then we have the representation

$$
X_{T}(F)=\bigcap_{\lambda \in \mathbf{C} \backslash F}(T-\lambda)^{p} X \quad \text { for all closed } F \subseteq \mathbf{C} .
$$

Moreover, if $S \in \mathscr{L}(X)$ is super-decomposable and satisfies $C(T, S)^{n} I=$ 0 for some $n \in \mathbf{N}$, then we have

$$
X_{S}(F)=\bigcap_{\lambda \in \mathbf{C} \backslash F}(S-\lambda)^{p+n-1} X \quad \text { for all closed } F \subseteq \mathbf{C} .
$$

1.2. Proposition. Let $T \in \mathscr{L}(X)$ be well-decomposable such that

$$
\bigcap_{\lambda \in \mathbf{C}, p \in \mathbf{N}}(T-\lambda)^{p} X=\{0\} .
$$

Then we have the algebraic representation

$$
X_{T}(F)=\bigcap_{\lambda \in \mathbf{C} \backslash F, p \in \mathbf{N}}(T-\lambda)^{p} X \quad \text { for all closed } F \subseteq \mathbf{C} .
$$

Moreover, if $S \in \mathscr{L}(X)$ is well-decomposable and satisfies $C(T, S)^{n} I=$ 0 for some $n \in \mathbf{N}$, then we have

$$
X_{S}(F)=\bigcap_{\lambda \in \mathbf{C} \backslash F, p \in \mathbf{N}}(S-\lambda)^{p} X \quad \text { for all closed } F \subseteq \mathbf{C} .
$$

The proofs of the main assertions (2) and (5) are quite similar and hence will be given simultaneously. The formally stronger statements (3) and (6) will then be deduced from (2) and (5), respectively. We shall need the following simple algebraic observation.

1.3. Lemma. Assume that the operators $A, S, T \in \mathscr{L}(X)$ satisfy $C(S, T)^{n} A=0$ for some $n \in \mathbf{N}$. Then we have

$$
A T^{p+n-1}(X) \subseteq S^{p}(X) \text { for all } p \in \mathbf{N}_{0} .
$$


Proof of 1.3. We proceed by induction. The case $p=0$ being trivial, let $p>0$ and assume that $A T^{q+n-1}(X) \subseteq S^{q}(X)$ holds for all $q \leq p$. Now, from $C(S, T)^{n} A=0$ we conclude that

$$
0=C(S, T)^{p+n} A=\sum_{k=0}^{p+n}\left(\begin{array}{c}
p+n \\
k
\end{array}\right) S^{k} A(-T)^{p+n-k},
$$

and consequently

$$
A T^{p+n}(X) \subseteq \sum_{k=1}^{p+n} S^{k} A T^{p+n-k}(X) .
$$

Next, for each $k=1, \ldots, p+n$ we observe that

$$
S^{k} A T^{p+n-k}(X) \subseteq S^{p+1}(X),
$$

which is obvious for $k \geq p+1$, and if $k<p+1$, it follows from our inductive hypothesis by taking $q=p-k+1$. We finally arrive at the inclusion

$$
A T^{p+n}(X) \subseteq S^{p+1}(X),
$$

thus completing the inductive argument. The assertion follows.

Proof of 1.1 and 1.2. Given an arbitrary closed subset $F$ of $\mathbf{C}$, let $G_{T}(F)$ denote either the intersection on the right-hand side of (2) or (5), respectively. Since it is well known that $X_{T}(F) \in \operatorname{Lat}(T)$ and $\sigma\left(T \mid X_{T}(F)\right) \subseteq F$, we obtain immediately $X_{T}(F) \subseteq G_{T}(F)$ both for 1.1 and 1.2. To prove the reverse inclusion, we shall show that

$$
G_{T}(F) \subseteq X_{T}(\bar{V}),
$$

where $V$ denotes an arbitrary open neighborhood of $F$ in $\mathbf{C}$. Given such an open set $V$, we choose another open set $U \subseteq \mathbf{C}$ such that $F \subseteq U \subseteq \bar{U} \subseteq V$ and observe that $\{V, \mathbf{C} \backslash \bar{U}\}$ is an open covering of $\mathbf{C}$. Since $T$ is well-decomposable, it follows that there exist an $R \in \mathscr{L}(X)$ and an integer $n \in \mathbf{N}$ such that

$$
C(T, T)^{n} R=0, \quad R(X) \subseteq X_{T}(\bar{V}), \quad(I-R)(X) \subseteq X_{T}(\mathbf{C} \backslash U) ;
$$

moreover, in the super-decomposable case we may take $n=1$, which amounts to $R T=T R$. Now, to show that $G_{T}(F)$ is contained in $X_{T}(\bar{V})$, it certainly suffices to prove that $(I-R) G_{T}(F)=\{0\}$. Because of our basic assumption (1) and (4), respectively, this assertion reduces to

$$
(I-R) G_{T}(F) \subseteq(T-\lambda)^{p} X
$$


where $\lambda \in \mathbf{C}$ is arbitrarily given and $p \in \mathbf{N}$ is either the integer given by condition (1) in case 1.1 or an arbitrary integer in case 1.2. Now, if $\lambda \in F$, the desired inclusion is obvious, since $(I-R) G_{T}(F)$ is contained in $X_{T}(\mathbf{C} \backslash U)$ and since the restriction $(T-\lambda) \mid X_{T}(\mathbf{C} \backslash U)$ is known to be invertible for each $\lambda \in U$ and hence in particular for each $\lambda \in F$. In the remaining case $\lambda \in \mathbf{C} \backslash F$, it follows from the definition that

$$
G_{T}(F) \subseteq(T-\lambda)^{p+n-1}(X)
$$

holds both for 1.1 and 1.2. Moreover, from $C(T, T)^{n} R=0$ we conclude that $C(T-\lambda, T-\lambda)^{n}(I-R)=0$ and therefore

$$
(I-R)(T-\lambda)^{p+n-1}(X) \subseteq(T-\lambda)^{p} X
$$

by the preceding Lemma 1.3. We thus arrive at $(I-R) G_{T}(F) \subseteq$ $(T-\lambda)^{p} X$, which completes the proof of (2) and (5). Now, to prove the remaining assertions, let $S \in \mathscr{L}(X)$ satisfy $C(T, S)^{n} I=0$ for some $n \in \mathbf{N}$. Then again Lemma 1.3 ensures that

$$
(S-\lambda)^{p+n-1}(X) \subseteq(T-\lambda)^{p}(X) \text { for all } \lambda \in \mathbf{C} .
$$

Consequently $S$ satisfies the corresponding version of condition (1) or (4) so that the final assertions (3) and (6) follow immediately from (2) and (5), respectively.

Using the same circle of ideas one can show that the algebraic representation of the spectral maximal spaces in the sense of (2) or (5) is preserved by nilpotent equivalence. Recall from [1] that two operators $S, T \in \mathscr{L}(X)$ are said to be nilpotent equivalent if $C(S, T)^{n} I=0=$ $C(T, S)^{n} I$ holds for some $n \in \mathbf{N}$. It is interesting to observe that the following result ceases to be true for the case of quasinilpotent equivalence in the sense of [4], since there are examples of quasinilpotent operators $T \in \mathscr{L}(X)$ which fail to satisfy condition (4); see for instance Remark 1.6 of [13].

1.4. Proposition. Let $T \in \mathscr{L}(X)$ be a decomposable operator such that condition (5) is fulfilled, and consider a nilpotent equivalent operator $S \in \mathscr{L}(X)$. Then $S$ is decomposable and satisfies condition (6). A similar result holds for the conditions (2) and (3).

Proof. Since nilpotent equivalence certainly implies quasinilpotent equivalence in the sense of [4], we infer from Theorem 2.2.1 of [4] that $S$ is also decomposable and satisfies $X_{S}(F)=X_{T}(F)$ for every 
closed $F \subseteq \mathbf{C}$. Now, if $n \in \mathbf{N}$ is chosen so that $C(T, S)^{n} I=0$, it follows from Lemma 1.3 and condition (5) that

$$
\begin{aligned}
X_{S}(F) & \subseteq \bigcap_{\lambda \notin F, p \in \mathbf{N}}(S-\lambda)^{p} X=\bigcap_{\lambda \notin F, p \in \mathbf{N}}(S-\lambda)^{p+n-1} X \\
& \subseteq \bigcap_{\lambda \notin F, p \in \mathbf{N}}(T-\lambda)^{p} X=X_{T}(F)=X_{S}(F) .
\end{aligned}
$$

We conclude that condition (6) is fulfilled. The proof of the final assertion is of course similar and therefore is omitted.

In $\S 3$, the preceding results will be applied to generalized scalar operators and to some other operators which admit a non-analytic functional calculus. We close this section with another typical application to multiplication operators and to multipliers on certain commutative Banach algebras.

1.5. EXAMPLE. Let $X$ be a commutative, semi-simple, and regular Banach algebra, and let $a \in X$. Then the operator $T \in \mathscr{L}(X)$ given by $T(x)=a x$ for all $x \in X$ is super-decomposable and satisfies

$$
X_{T}(F)=\bigcap_{\lambda \in \mathbf{C} \backslash F}(T-\lambda) X \text { for all closed } F \subseteq \mathbf{C} .
$$

1.6. EXAMPLE. Let $G$ be a locally compact abelian group, and consider a regular Borel measure $\mu \in M(G)$, whose continuous part is absolutely continuous. Then the operator $T \in \mathscr{L}(X)$, given by convolution with $\mu$ either on $X=L^{1}(G)$ or on $X=M(G)$, is superdecomposable and satisfies

$$
X_{T}(F)=\bigcap_{\lambda \in \mathbf{C} \backslash F}(T-\lambda) X \quad \text { for all closed } F \subseteq \mathbf{C} .
$$

Proof of 1.5 and 1.6. We first note that every multiplication operator $T$ on a commutative Banach algebra $X$ satisfies

$$
\bigcap_{\lambda \in \mathbf{C}}(T-\lambda) X \subseteq \operatorname{rad}(X)
$$

the radical of $X$, so that this intersection is trivial whenever $X$ is semisimple. We conclude that the operators considered in 1.5 and 1.6 satisfy condition (1) with $p=1$. On the other hand, these operators are super-decomposable by Corollary 2.4 and Theorem 2.5 of [13]. Hence the assertions follow immediately from Proposition 1.1. 
2. Preliminaries on differentiable functions. In the present section, we collect some auxiliary results on partitions of unity and norm estimates for differentiable functions. Let $n \in \mathbf{N}$ be given, and endow $\mathbf{R}^{n}$ with the Euclidean metric. For $K \subseteq \mathbf{R}^{n}$ and $\varepsilon>0$, let $K(\varepsilon):=\left\{x \in \mathbf{R}^{n}: \operatorname{dist}(x, K) \leq \varepsilon\right\}$. Given a mult-index $\alpha \in \mathbf{N}_{0}^{n}$, let

$$
|\alpha|:=\alpha_{1}+\cdots+\alpha_{n} \text { and } D^{\alpha}:=\partial_{1}^{\alpha_{1}} \cdots \partial_{n}^{\alpha_{n}},
$$

where $\partial_{1}, \ldots, \partial_{n}$ denote the usual operators of partial differentiation. Finally, let $\|\cdot\|_{\infty}$ denote the supremum norm for bounded complexvalued functions on $\mathbf{R}^{n}$. Our first result is a slight extension of a standard result in calculus; see for instance [14].

2.1. Proposition. For each $r \in \mathbf{N}$ and all $\alpha \in \mathbf{N}_{0}^{n}$ there exists $a$ constant $c_{\alpha}>0$ with the following property: given any collection of compact non-empty subsets $K_{1}, \ldots, K_{r}$ of $\mathbf{R}^{n}$ and $\varepsilon>0$, there exist $e_{1}, \ldots, e_{r} \in \mathscr{C}^{\infty}\left(\mathbf{R}^{n}\right)$ such that the following conditions are fulfilled:

(9) $e_{1}+\cdots+e_{r} \equiv 1$ on some neighborhood of $K_{1} \cup \cdots \cup K_{r}$,

$$
\begin{gathered}
0 \leq e_{j} \leq 1 \text { for } j=1, \ldots, r, \\
\operatorname{supp} e_{j} \subseteq K_{j}(\varepsilon) \text { for } j=1, \ldots, r \\
+\cdots+e_{r} \equiv 1 \text { on some neighborhood of } K_{1} \cup \cdots \cup K_{r} \\
\left\|D^{\alpha} e_{j}\right\|_{\infty} \leq c_{\alpha} \varepsilon^{-|\alpha|} \text { for all } \alpha \in \mathbf{N}_{0}^{n} \text { and } j=1, \ldots, r .
\end{gathered}
$$

Proof. For $r=1$ the assertion follows immediately from Lemma I.4.2 of [14]. Let $c_{\alpha}>0$ for $\alpha \in \mathbf{N}_{0}^{n}$ denote the constants corresponding to the case $r=1$. Fix an arbitrary $r \in \mathbf{N}$, and consider compact nonempty sets $K_{1}, \ldots, K_{r} \subseteq \mathbf{R}^{n}$ and some $\varepsilon>0$. Then, for every $j=$ $1, \ldots, r$ we obtain some $f_{j} \in \mathscr{C}^{\infty}\left(\mathbf{R}^{n}\right)$ such that $0 \leq f_{j} \leq 1, \operatorname{supp} f_{j} \subseteq$ $K_{j}(\varepsilon), f_{j} \equiv 1$ on a neighborhood of $K_{j}$, and

$$
\left\|D^{\alpha} f_{j}\right\|_{\infty} \leq c_{\alpha} \varepsilon^{-|\alpha|} \text { for all } \alpha \in \mathbf{N}_{0}^{n} .
$$

Obviously, the functions $e_{1}, \ldots, e_{r} \in \mathscr{C}^{\infty}\left(\mathbf{R}^{n}\right)$ given by

$$
\begin{aligned}
e_{1}= & f_{1}, \\
e_{2}= & \left(1-f_{1}\right) f_{2}, \\
& \vdots \\
e_{r}= & \left(1-f_{1}\right) \cdots\left(1-f_{r-1}\right) f_{r}
\end{aligned}
$$

satisfy (7) and (8). Moreover, one easily verifies by induction that

$$
e_{1}+\cdots+e_{r}=1-\left(1-f_{1}\right) \cdots\left(1-f_{r}\right),
$$


from which we conclude that condition (9) is fulfilled as well. Finally, let $\alpha \in \mathbf{N}_{0}^{n}$ be given arbitrarily. Then for all functions $u$ and $v$ of the form $f_{i}$ or $1-f_{j}$ we obtain from the Leibniz rule

$$
\begin{aligned}
\left\|D^{\alpha}(u v)\right\|_{\infty} & =\left\|\sum_{\beta \leq \alpha}\left(\begin{array}{l}
\alpha \\
\beta
\end{array}\right) D^{\alpha-\beta}(u) D^{\beta}(v)\right\|_{\infty} \\
& \leq \sum_{\beta \leq \alpha}\left(\begin{array}{l}
\alpha \\
\beta
\end{array}\right) c_{\alpha-\beta} \varepsilon^{-|\alpha|+|\beta|} c_{\beta} \varepsilon^{-|\beta|}=\check{c}_{\alpha} \varepsilon^{-|\alpha|}
\end{aligned}
$$

for a suitable new constant $\check{c}_{\alpha}>0$. An obvious repetition of this argument shows that the estimates in (10) are fulfilled for a suitable choice of constants. The assertion follows.

For fixed $k \in \mathbf{N}_{0}$ it will be convenient to introduce

$$
M=\max \left\{1, c_{\alpha}: \alpha \in \mathbf{N}_{0}^{n} \text { with }|\alpha| \leq k\right\},
$$

where $c_{\alpha}$ are the constants from Proposition 2.1. This choice may illuminate the assumption in the following result.

2.2. Proposition. Let $k \in \mathbf{N}_{0}$ and $M \geq 1$ be given, and consider $e_{m} \in \mathscr{C}^{\infty}\left(\mathbf{R}^{n}\right)$ and $\varepsilon_{m}>0$ for all $m \in \mathbf{N}$ such that the following two conditions are fulfilled:

$$
\begin{gathered}
\left\|e_{m}\right\|_{\infty} \leq 1 \text { for all } m \in \mathbf{N} \\
\left\|D^{\alpha} e_{m}\right\|_{\infty} \leq M \varepsilon_{m}^{-|\alpha|} \text { for all } m \in \mathbf{N}, \alpha \in \mathbf{N}_{0}^{n} \text { with }|\alpha| \leq k
\end{gathered}
$$

Then for all $m \in \mathbf{N}$ and $\alpha \in \mathbf{N}_{0}^{n}$ with $|\alpha| \leq k$ we have:

$$
\left\|D^{\alpha}\left(e_{1} \cdots e_{m}\right)\right\|_{\infty} \leq M^{|\alpha|}\left(\frac{1}{\varepsilon_{1}}+\cdots+\frac{1}{\varepsilon_{m}}\right)^{|\alpha|} .
$$

Proof. For notational convenience, we restrict ourselves to the case $n=2$, which should be sufficiently typical and is all we need in the following. So let us consider an $m \in \mathbf{N}$ and an $\alpha=(p, q)$, where $p, q \in \mathbf{N}_{0}$ and $|\alpha|=p+q \leq k$. Then we have:

$$
\begin{aligned}
D^{\alpha}\left(e_{1} \cdots e_{m}\right)=\partial_{2}^{q} \partial_{1}^{p}\left(e_{1} \cdots e_{m}\right) \\
=\partial_{2}^{q}\left(\sum_{l_{1}=1}^{m} \cdots \sum_{l_{p}=1}^{m} e_{1} \cdots \partial_{1} e_{l_{1}} \cdots \partial_{1} e_{l_{p}} \cdots e_{m}\right) \\
=\sum_{l_{1}=1}^{m} \cdots \sum_{l_{p}=1}^{m} \sum_{k_{1}=1}^{m} \cdots \sum_{k_{q}=1}^{m} e_{1} \cdots \partial_{1} e_{l_{1}} \cdots \partial_{1} e_{l_{p}} \cdots \partial_{2} e_{k_{1}} \cdots \partial_{2} e_{k_{q}} \cdots e_{m} .
\end{aligned}
$$


Here we adopt an obvious convention concerning higher derivatives whenever $l_{i}=l_{j}, k_{i}=k_{j}$, or $l_{i}=k_{j}$. Now observe that $M \geq 1$ and $p+q=|\alpha|$. Hence, with the appropriate case for higher derivatives, we conclude from our assumptions:

$$
\begin{aligned}
& \left\|D^{\alpha}\left(e_{1} \cdots e_{m}\right)\right\|_{\infty} \\
& \quad \leq M^{p+q} \sum_{l_{1}=1}^{m} \cdots \sum_{l_{p}=1}^{m} \sum_{k_{1}=1}^{m} \cdots \sum_{k_{q}=1}^{m} \frac{1}{\varepsilon_{l_{1}}} \cdots \frac{1}{\varepsilon_{l_{p}}} \frac{1}{\varepsilon_{k_{1}}} \cdots \frac{1}{\varepsilon_{k_{q}}} \\
& \quad=M^{p+q}\left(\frac{1}{\varepsilon_{1}}+\cdots+\frac{1}{\varepsilon_{m}}\right)^{p+q}=M^{|\alpha|}\left(\frac{1}{\varepsilon_{1}}+\cdots+\frac{1}{\varepsilon_{m}}\right)^{|\alpha|}
\end{aligned}
$$

The assertion follows.

2.3. Corollary. Let $k \in \mathbf{N}_{0}, M \geq 1,0<\delta<1$, and $e_{m} \in \mathscr{C}^{\infty}\left(\mathbf{R}^{n}\right)$ such that $\left\|e_{m}\right\|_{\infty} \leq 1$ for all $m \in \mathbf{N}$. Assume that

$$
\left\|D^{\alpha} e_{m}\right\|_{\infty} \leq M \delta^{-m|\alpha|} \text { for all } m \in \mathbf{N}, \alpha \in \mathbf{N}_{0}^{n} \text { with }|\alpha| \leq k .
$$

Then there exists a constant $\tilde{M} \geq 1$ such that

$$
\left\|D^{\alpha}\left(e_{1} \cdots e_{m}\right)\right\|_{\infty} \leq \tilde{M} \delta^{-m|\alpha|} \text { for all } m \in \mathbf{N}, \alpha \in \mathbf{N}_{0}^{n} \text { with }|\alpha| \leq k .
$$

Proof. The assertion follows immediately from Proposition 2.2 with the obvious choice $\varepsilon_{m}=\delta^{m}$ for all $m \in \mathbf{N}$.

3. Main results. We turn now to the algebraic representation of the spectral maximal spaces for certain operators which admit a functional calculus on a suitable algebra of functions. It will follow from [13] that all the operators to be considered here are super-decomposable so that the general reduction theory from $\S 1$ may be applied. We start with the most prominent case, that of generalized scalar operators.

Given a complex Banach space $X$, an operator $T \in \mathscr{L}(X)$ is called generalized scalar [4], [22], if there exists a continuous homomorphism $\Phi: \mathscr{C}^{\infty}(\mathbf{C}) \rightarrow \mathscr{L}(X)$ satisfying $\Phi(1)=I$ and $\Phi(Z)=T$, where $Z$ denotes the identity function on $\mathbf{C}$. Let us mention in passing that the continuity of such a $\mathscr{C}^{\infty}$-functional calculus $\Phi$ for $T$ is equivalent to a certain algebraic condition on $T$, namely that every linear subspace $Y$ of $X$ which is $T$-divisible in the sense of $(T-\lambda) Y=Y$ for all $\lambda \in \mathbf{C}$ has to be zero; see Theorem 6.5 of [2]. Of course, the continuity of $\Phi: \mathscr{C}^{\infty}(\mathbf{C}) \rightarrow \mathscr{L}(X)$ means precisely that there exist some compact $\Omega \subseteq \mathbf{C}$, a constant $c \geq 0$, and an integer $k \geq 0$ such that

$$
\|\Phi(f)\| \leq c\|f\|_{k, \Omega} \text { for all } f \in \mathscr{C}^{\infty}(\mathbf{C}),
$$


where $\mathbf{C}$ is canonically identified with $\mathbf{R}^{2}$, and the seminorm $\|\cdot\|_{k, \Omega}$ on $\mathscr{C}^{\infty}(\mathbf{C})$ is given by

$$
\|f\|_{k, \Omega}=\sum_{\alpha \in \mathbf{N}_{0}^{2},|\alpha| \leq k} \frac{1}{\alpha !} \sup \left\{\left|D^{\alpha} f(z)\right|: z \in \Omega\right\}
$$

for all $f \in \mathscr{C}^{\infty}(\mathbf{C})$. The order $k(T) \in \mathbf{N}_{0}$ of a generalized scalar operator $T \in \mathscr{L}(X)$ is defined to be the smallest integer $k \geq 0$ such that (11) holds for some $\mathscr{C}^{\infty}$-functional calculus $\Phi$ for $T$. Note that every generalized scalar operator is super-decomposable by Proposition 2.2 of [13].

3.1. THEOREM. If $T \in \mathscr{L}(X)$ is generalized scalar, then for all closed $F \subseteq \mathbf{C}$ and all $p \in \mathbf{N}$ with $p \geq k(T)+3$ we have

$$
X_{T}(F)=\bigcap_{\lambda \in \mathbf{C} \backslash F}(T-\lambda)^{p} X .
$$

Proof. In view of Proposition 1.1, it suffices to show that if

$$
y \in \bigcap_{\lambda \in \mathbf{C}}(T-\lambda)^{p} X \quad \text { for some } p \geq k(T)+3,
$$

then necessarily $y=0$. Choose a $\mathscr{C}^{\infty}$-functional calculus $\Phi$ for $T$ such that (11) holds for some compact $\Omega \subseteq \mathbf{C}$ and $k=k(T)$, and let $e_{0} \in \mathscr{C}^{\infty}(\mathbf{C})$ have compact support and satisfy $0 \leq e_{0} \leq 1$ and $e_{0} \equiv 1$ on some neighborhood of $\Omega$. Then it is clear that $\Phi\left(e_{0}\right) y=y$. Next, choose a square $P_{0} \subseteq \mathrm{C}$ such that supp $e_{0} \subseteq P_{0}$ and fix an arbitrary constant $\gamma, 0<\gamma \leq \frac{1}{8}$. Define $\delta=\frac{1}{2}+2 \gamma$ and $\varepsilon_{n}=\gamma \delta^{n-1} s\left(P_{0}\right)$ for all $n \in \mathbf{N}$, where $s(P)$ denotes the side length of a square $P \subseteq \mathbf{C}$. Now divide $P_{0}$ into four squares $K_{1}, \ldots, K_{4}$ with size length $\frac{1}{2} s\left(P_{0}\right)$ and apply Proposition 2.1 with the choice $\varepsilon=\varepsilon_{1}$. For the corresponding partition of unity $f_{1}, \ldots, f_{4}$ in $\mathscr{C}^{\infty}(\mathbf{C})$ we obtain the estimate

$$
\|y\|=\left\|\Phi\left(e_{0}\right) y\right\|=\left\|\Phi\left(\sum_{j=1}^{4} f_{j} e_{0}\right) y\right\| \leq \sum_{j=1}^{4}\left\|\Phi\left(f_{j} e_{0}\right) y\right\|,
$$

and consequently $\|y\| \leq 4\left\|\Phi\left(f_{j} e_{0}\right) y\right\|=4\left\|\Phi\left(f_{j}\right) y\right\|$ for at least one $j \in$ $\{1, \ldots, 4\}$. Let $e_{1}=f_{j}$ and $P_{1}=K_{j}\left(\varepsilon_{1}\right)$ for such a $j \in\{1, \ldots, 4\}$. Then we have $e_{1} \in \mathscr{C}^{\infty}(\mathbf{C})$ and $0 \leq e_{1} \leq 1, \operatorname{supp} e_{1} \subseteq P_{1},\|y\| \leq 4\left\|\Phi\left(e_{1}\right) y\right\|$, and

$$
\left\|D^{\alpha} e_{1}\right\|_{\infty} \leq M \varepsilon_{1}^{-|\alpha|} \text { for all } \alpha \in \mathbf{N}_{0}^{2} \text { with }|\alpha| \leq k,
$$

where $M \geq 1$ is some universal constant. Moreover, it is obvious that $s\left(P_{1}\right)=\delta s\left(P_{0}\right)$. Proceeding in the same way by induction, we obtain 
for each $n \in \mathbf{N}$ a function $e_{n} \in \mathscr{C}^{\infty}(\mathbf{C})$ and a square $P_{n} \subseteq \mathbf{C}$ such that the following conditions are fulfilled:

$$
\begin{gathered}
0 \leq e_{n} \leq 1 ; \quad \operatorname{supp} e_{n} \subseteq P_{n} ; \quad s\left(P_{n}\right)=\delta^{n} s\left(P_{0}\right) ; \\
\left\|D^{\alpha} e_{n}\right\|_{\infty} \leq M \varepsilon_{n}^{-|\alpha|} \quad \text { for all } \alpha \in \mathbf{N}_{0}^{2} \text { with }|\alpha| \leq k ; \\
\|y\| \leq 4\left\|\Phi\left(e_{1}\right) y\right\| \leq \cdots \leq 4^{n}\left\|\Phi\left(e_{1} \cdots e_{n}\right) y\right\| .
\end{gathered}
$$

Since the intersection of the squares $P_{n}$ may well be empty, we finally introduce for each $n \in \mathbf{N}_{0}$ the square $Q_{n}$ with the same center as $P_{n}$, but with twice the side length of $P_{n}$. Then one easily deduces from the assumption $\gamma \leq \frac{1}{8}$ that $Q_{n} \subseteq Q_{n-1}$ for all $n \in \mathbf{N}$. Since $s\left(Q_{n}\right) \rightarrow 0$ as $n \rightarrow \infty$, it follows that the squares $Q_{n}$ decrease to exactly one point

$$
\lambda \in \bigcap_{n=1}^{\infty} Q_{n} .
$$

Now, by our assumption on $y$ there exists some $x \in X$ such that $y=(T-\lambda)^{p} x$. Since supp $e_{n} \subseteq P_{n} \subseteq Q_{n}$, we arrive at the estimates

$$
\begin{aligned}
\|y\| & \leq 4^{n}\left\|\Phi\left(e_{1} \cdots e_{n}(Z-\lambda)^{p}\right) x\right\| \\
& \leq 4^{n} c\|x\|\left\|e_{1} \cdots e_{n}(Z-\lambda)^{p}\right\|_{k, Q_{n}} \\
& =4^{n} c\|x\| \sum_{|\alpha| \leq k} \frac{1}{\alpha !}\left\|D^{\alpha}\left(e_{1} \cdots e_{n}(Z-\lambda)^{p}\right)\right\|_{0, Q_{n}} \\
& \leq 4^{n} c\|x\| \sum_{|\alpha| \leq k} \frac{1}{\alpha !} \sum_{\beta \leq \alpha}\left(\begin{array}{c}
\alpha \\
\beta
\end{array}\right)\left\|D^{\alpha-\beta}\left(e_{1} \cdots e_{n}\right)\right\|_{\infty}\left\|D^{\beta}(Z-\lambda)^{p}\right\|_{0, Q_{n}}
\end{aligned}
$$

for all $n \in \mathbf{N}$. The last terms can be estimated as follows. Since $\lambda \in Q_{n}$ and $s\left(Q_{n}\right)=2 \delta^{n} s\left(P_{0}\right)$, an elementary calculation shows that

$$
\left\|D^{\beta}(Z-\lambda)^{p}\right\|_{0, Q_{n}} \leq c_{1} \delta^{n(p-|\beta|)} \text { for all } n \in \mathbf{N}
$$

with some suitable constant $c_{1} \geq 0$ not depending on $n$. On the other hand, it follows from the choice of $\varepsilon_{n}$ and from Corollary 2.3 that

$$
\left\|D^{\alpha-\beta}\left(e_{1} \cdots e_{n}\right)\right\|_{\infty} \leq c_{2} \delta^{-n(|\alpha|-|\beta|)} \quad \text { for all } n \in \mathbf{N},
$$

where again the constant $c_{2} \geq 0$ does not depend on $n$. Taking all these estimates into account, we finally obtain

$$
\|y\| \leq c_{3} 4^{n} \delta^{n p-n k}=c_{3}\left(4 \delta^{p-k}\right)^{n} \quad \text { for all } n \in \mathbf{N}
$$

for some suitable constant $c_{3} \geq 0$ not depending on $n$. Recall that $p-k \geq 3$ and that $\delta=\frac{1}{2}+2 \gamma$, where $0<\gamma \leq \frac{1}{8}$ could have been chosen arbitrarily small. Hence it is possible to achieve $4 \delta^{3}<1$ for 
a suitable choice of $\gamma$. Letting $n \rightarrow \infty$ in the last inequality for $y$, we conclude that $y=0$, which completes the proof.

3.2. REMARKS. (a) The last lines of the preceding proof for the representation (12) reveal that the best possible exponent $p$ which can be obtained by the present approach is $p=k(T)+3$. Exactly the same exponent for the representation (12) was also obtained by Foias and Vasilescu in Theorem 3.2 of [8] by using a completely different method. On the other hand, we shall see that in certain cases the optimal exponent for (12) can be improved to be $p=k(T)+2$. Let us note that in general the representation (12) need not hold for $p<$ $k(T)+2$, as can be easily derived from Example 2.10 of [8] even in the case of certain spectral operators.

(b) If the operator $T \in \mathscr{L}(X)$ has a functional calculus on $\mathscr{C}^{\infty}(\mathbf{R})$, then our method can be carried through by using intervals in $\mathbf{R}$ instead of squares in $\mathbf{C}$. In this case, the respective interval will be divided into just two subintervals in each inductive step. This modification will obviously result in the weaker inequality $2 \delta^{p-k}<1$ instead of $4 \delta^{p-k}<1$, which can be fulfilled by some $\delta>\frac{1}{2}$ provided that $p-k \geq$ 2. Hence, in this case, our optimal exponent for (12) turns out to be $p=k(T)+2$. This was also observed in [8] using a different type of argument.

(c) If the operator $T \in \mathscr{L}(X)$ has a $\mathscr{C}^{\infty}(\mathbf{C})$-functional calculus of order $k(T)$ and if $\sigma(T) \subseteq \mathbf{R}$, then it is shown in Theorem 5.4.5 of [4] that $T$ admits a $\mathscr{C}^{\infty}(\mathbf{R})$-functional calculus of order $\leq 2 k(T)$. Using the improved result of (b), this observation will lead to a better exponent in the case $k(T)=0$, but not necessarily in the case $k(T) \geq 1$.

(d) The geometry of squares is less important for our approach to the representation (12) than one might expect. For instance, one may exploit the same kind of argument with triangles instead of squares in the complex plane, where at each step of the inductive construction the respective triangle will have to be divided into two subtriangles of equal size. This modification of the preceding proof leads to the representation (12) with exactly the same optimal exponent $p=k(T)+3$; let us note that the approach given in [23] is based on triangles instead of squares.

(e) Since hyponormal operators are known to be, up to similarity, restrictions of generalized scalar operators [18], an interesting consequence of Theorem 3.1 is that hyponormal and, in particular, subnormal operators do not have non-zero divisible subspaces. This observation is of particular importance in the automatic continuity theory 
for intertwining linear transformations; see for instance [9], [12], [13], [15], [21].

(f) Finally, let $T=\left(T_{1}, \ldots, T_{n}\right)$ be a system of several commuting operators from $\mathscr{L}(X)$, and assume that the system $T$ has a continuous functional calculus on $C^{\infty}\left(\mathbf{C}^{n}\right)$. Then an obvious extension of our approach to Theorem 3.1 will lead precisely to the algebraic representation of the spectral maximal spaces for the system $T$ in the sense of Corollary 3.7 of [3]. In this case, the best possible exponent given by our method turns out to be $p=k(T)+2 n+1$, which was also obtained by Albrecht and Vasilescu [3] by expanding the corresponding argument from [8]. This higher dimensional result is also closely related to Theorem 3.3 of [10].

We now turn to the case of functional calculi on certain Lipschitz algebras. Given an arbitrary $\alpha, 0<\alpha<1$ and a nonempty compact subset $K$ of $\mathbf{C}$, let $\operatorname{Lip}_{\alpha}(K)$ denote the space of all functions $f: K \rightarrow \mathbf{C}$ such that

$$
p_{\alpha}(f)=\sup \left\{\frac{|f(u)-f(v)|}{|u-v|^{\alpha}}: u, v \in K \text { with } u \neq v\right\}<\infty .
$$

It is well known and easily seen that $\operatorname{Lip}_{\alpha}(K)$ is a regular Banach function algebra with respect to pointwise multiplication, where the norm is given by $\|\cdot\|_{\alpha}=\|\cdot\|_{\infty}+p_{\alpha}$ on $\operatorname{Lip}_{\alpha}(K)$. Hence, if $\Phi: \operatorname{Lip}_{\alpha}(K) \rightarrow$ $\mathscr{L}(X)$ is a continuous homomorphism with $\Phi(1)=I$, then for each $f \in \operatorname{Lip}_{\alpha}(K)$ the operator $\Phi(f) \in \mathscr{L}(X)$ is super-decomposable by Theorem 2.3 of [13]. Moreover, the operator $T=\Phi(Z)$ is obviously generalized scalar with $k(T) \leq 1$ so that Theorem 3.1 yields the representation (12) with $p \geq 4$. Actually this can be improved as follows.

3.3. THEOREM. Let $\Phi: \operatorname{Lip}_{\alpha}(K) \rightarrow \mathscr{L}(X)$ be a continuous unital homomorphism, where $0<\alpha<1$ and $K \subseteq \mathbf{C}$ is compact. Then the operator $T=\Phi(Z) \in \mathscr{L}(X)$ satisfies (12) for all $p \geq 3$. Moreover if $K \subseteq \mathbf{R}$, then (12) holds for all $p \geq 2$.

Proof. Given an arbitrary $p \in \mathbf{N}$, an obvious adaptation of the proof of Theorem 3.1 to the present situation leads to the following estimates

$$
\begin{aligned}
\|y\| & \leq 4^{n} c\|x\|\left\|e_{n} \cdots e_{1}(Z-\lambda)^{p}\right\|_{\alpha} \\
& \leq 4^{n} c\|x\|\left(\left\|(Z-\lambda)^{p}\right\|_{0, Q_{n}}+p_{\alpha}\left(f_{n}(Z-\lambda)^{p}\right)\right),
\end{aligned}
$$

where $f_{n}=e_{n} \cdots e_{1}$ on $K$ for all $n \in \mathbf{N}$. Since $s\left(Q_{n}\right)=2 \delta^{n} s\left(P_{0}\right)$ for some $\delta, \frac{1}{2}<\delta<1$, we obtain immediately

$$
\left\|(Z-\lambda)^{p}\right\|_{0, Q_{n}} \leq c_{1} \delta^{n p} \quad \text { for all } n \in \mathbf{N}
$$


and for some constant $c_{1} \geq 0$ not depending on $n$. To estimate the term involving $p_{\alpha}$ let us introduce

$$
H_{n}(u, v)=\frac{\left|f_{n}(u)(u-\lambda)^{p}-f_{n}(v)(v-\lambda)^{p}\right|}{|u-v|^{\alpha}}
$$

for all $n \in \mathbf{N}$ and all $u, v \in K$ with $u \neq v$. If both $u, v \notin P_{n}$, we infer from supp $f_{n} \subseteq P_{n}$ that $f_{n}(u)=f_{n}(v)=0$ and hence $H_{n}(u, v)=0$. Without loss of generality, we may therefore assume that $u \in P_{n}$. Now, if $v \in K$ satisfies $|u-v|>s\left(Q_{n}\right)$, then obviously $v \notin P_{n}$ and hence $f_{n}(v)=0$, which implies that

$$
H_{n}(u, v) \leq s\left(Q_{n}\right)^{p-\alpha} \leq c_{1} \delta^{n(p-\alpha)} \quad \text { for all } n \in \mathbf{N} .
$$

It remains to estimate $H_{n}(u, v)$ for the case $u \in P_{n}$ and $|u-v| \leq s\left(Q_{n}\right)$. Then it is clear that $|u-\lambda| \leq s\left(Q_{n}\right)$ and $|v-\lambda| \leq 2 s\left(Q_{n}\right)$. Furthermore, since $\left\|D^{\beta} f_{n}\right\|_{\infty} \leq c_{2} \delta^{-n}$ for all $n \in \mathbf{N}$ and all $\beta \in \mathbf{N}_{0}^{2}$ with $|\beta| \leq 1$, we obtain

$$
\begin{aligned}
H_{n}(u, v) & \leq \frac{\left|f_{n}(u)-f_{n}(v)\right|}{|u-v|^{\alpha}}|u-\lambda|^{p}+\left|f_{n}(v)\right| \frac{\left|(u-\lambda)^{p}-(v-\lambda)^{p}\right|}{|u-v|^{\alpha}} \\
& \leq c_{3} \delta^{n(p-\alpha)}+\frac{\left|(u-\lambda)^{p}-(v-\lambda)^{p}\right|}{|u-v|^{\alpha}}
\end{aligned}
$$

for all $n \in \mathbf{N}$, where again the constants $c_{2}, c_{3} \geq 0$ do not depend on $n$. Finally observe that the last term can be estimated easily by making use of the identity

$$
a^{p}-b^{p}=(a-b)\left(a^{p-1}+a^{p-2} b+\cdots+a b^{p-2}+b^{p-1}\right)
$$

with the obvious choice $a=u-\lambda$ and $b=v-\lambda$. We conclude that

$$
H_{n}(u, v) \leq c_{4} \delta^{n(p-\alpha)} \quad \text { for all } n \in \mathbf{N},
$$

and consequently

$$
p_{\alpha}\left(f_{n}(Z-\lambda)^{p}\right) \leq c_{4} \delta^{n(p-\alpha)} \text { for all } n \in \mathbf{N}
$$

with some suitable constant $c_{4} \geq 0$ not depending on $n$. We finally arrive at

$$
\|y\| \leq c_{5}\left(4 \delta^{p-\alpha}\right)^{n} \quad \text { for all } n \in \mathbf{N}
$$

with some universal constant $c_{5} \geq 0$. Now, if we assume that $p \geq 3$, then choosing $\delta>\frac{1}{2}$, we have $4 \delta^{p-\alpha}<1$. Taking the limit as $n \rightarrow \infty$ in the last inequality for $y$, we conclude that $y=0$, which completes the proof of (12) for $p \geq 3$. In the special case $K \subseteq \mathbf{R}$, the proof can be carried through by using intervals in $\mathbf{R}$ instead of squares in $\mathbf{C}$. In this case, we arrive at the condition $2 \delta^{p-\alpha}<1$, which can be fulfilled 
by a suitable choice of $\delta>\frac{1}{2}$ provided that $p \geq 2$. This completes the proof.

The preceding result can be extended easily to the case of differentiable functions. For simplicity, let $I$ be a compact interval in $\mathbf{R}$, and consider some $k \in \mathbf{N}_{0}$ and some $\alpha, 0<\alpha<1$. Then $\mathscr{C}^{k, \alpha}(I)$ is defined to be the space of all $k$ times continuously differentiable functions $f: I \rightarrow \mathbf{C}$ with the property $f^{(k)} \in \operatorname{Lip}_{\alpha}(I)$. With the usual pointwise operations, $\mathscr{C}^{k, \alpha}(I)$ is a regular Banach algebra with respect to a suitable algebra norm which is equivalent to the norm $\|\cdot\|_{k, \alpha}$ given by

$$
\|f\|_{k, \alpha}=\sum_{j=0}^{k}\left\|f^{(j)}\right\|_{\infty}+p_{\alpha}\left(f^{(k)}\right) \quad \text { for all } f \in \mathscr{C}^{k, \alpha}(I) .
$$

The proof of the following result is similar to the proofs of 3.1 and 3.3 and is therefore omitted.

3.4. THEOREM. Let $\Phi: \mathscr{C}^{k, \alpha}(I) \rightarrow \mathscr{L}(X)$ be a continuous unital homomorphism, where $k \in \mathbf{N}_{0}, 0<\alpha<1$, and $I \subseteq \mathbf{R}$ is a compact interval. Then the operator $T=\Phi(Z) \in \mathscr{L}(X)$ satisfies (12) for all $p \geq k+2$.

There are some other interesting cases, where the optimal exponent $p$ for the representation (12) given by 3.1 can be improved. For example, let $A C(I)$ denote the Banach algebra of all absolutely continuous functions $f: I \rightarrow \mathbf{C}$ for a compact interval $I \subseteq \mathbf{R}$, where the norm $\|\cdot\|$ is given by

$$
\|f\|:=\|f\|_{\infty}+\int_{I}\left|f^{\prime}(t)\right| d t \quad \text { for all } f \in A C(I) .
$$

Then, for every continuous unital homomorphism $\Phi: A C(I) \rightarrow \mathscr{L}(X)$, the operator $T=\Phi(Z) \in \mathscr{L}(X)$ satisfies (12) for all $p \geq 2$. This can be seen following the lines of the proof of Theorem 3.1, but the details are slightly more involved and will not be given here.

In light of the preceding results, one might conjecture that every operator, which admits a continuous functional calculus on a reasonable algebra of functions with partitions of unity, should enjoy the algebraic representation of the spectral maximal spaces in the sense of (12) for some suitable integer $p$. The following counterexample will show, however, that in general such operators may have divisible subspaces different from zero and hence will fail to satisfy even condition (4). The basic idea is to look for a suitable quasinilpotent 
operator, since such an operator cannot be generalized scalar unless it is nilpotent; see for instance Proposition 4.1 of [15]. Actually, the existence of non-trivial divisible subspaces rules out even the weak kind of algebraic representation of the spectral maximal spaces considered in Proposition 1.5 of [13].

3.5. EXAMPLE. There exists a compact and quasinilpotent operator $T \in \mathscr{L}(X)$ on a Banach space $X$, such that $T$ has a non-trivial divisible subspace, but admits a continuous functional calculus on a regular natural Banach function algebra contained in $\mathscr{C}^{\infty}([0,1])$.

Proof. Consider the Banach space $X=\mathscr{C}([0,1])$ and the Volterra operator $T \in \mathscr{L}(X)$ given by

$$
(T f)(s)=\int_{0}^{s} f(t) d t \quad \text { for all } f \in \mathscr{C}([0,1]) \text { and } s \in[0,1] \text {. }
$$

Then $T$ is both compact and quasinilpotent and has the following non-trivial divisible subspace

$$
Y=\left\{f \in \mathscr{C}^{\infty}([0,1]): f^{(k)}(0)=0 \text { for all } k \in \mathbf{N}_{0}\right\} .
$$

Next, take some $\alpha, 1<\alpha<2$ and consider the space $A_{\alpha}$ consisting of all $f \in \mathscr{C}^{\infty}([0,1])$ with the property

$$
\|f\|_{\alpha}=\sum_{k=0}^{\infty} \frac{\left\|f^{(k)}\right\|_{\infty}}{(k !)^{\alpha}}<\infty .
$$

This space has been considered in a number of articles; in particular, it follows from the results in [5] and [16] that the space $A_{\alpha}$ endowed with the norm $\|\cdot\|_{\alpha}$ is a Banach algebra with the maximal ideal space $[0,1]$ and that the polynomials are dense in $A_{\alpha}$ with respect to the norm $\|\cdot\|_{\alpha}$. Moreover, one can use the Denjoy-Carleman theorem and the standard techniques of the theory of non-quasianalytic classes to show that the Banach algebra $A_{\alpha}$ is regular and hence admits partitions of unity; in this connection we refer to Chapter 19 of [20]. Now, given a polynomial

$$
p(z)=\sum_{k=0}^{n} a_{k} z^{k}=\sum_{k=0}^{n} \frac{p^{(k)}(0)}{k !} z^{k} \quad \text { for all } z \in[0,1],
$$

we define $\Phi(p)=p(T) \in \mathscr{L}(X)$ and observe that

$$
\|\Phi(p)\| \leq \sum_{k=0}^{n} \frac{\left|p^{(k)}(0)\right|}{k !}\left\|T^{k}\right\| \leq \sum_{k=0}^{n} \frac{\left\|p^{(k)}\right\|_{\infty}}{(k !)^{\alpha}}(k !)^{\alpha-2}
$$


and hence $\|\Phi\|(p)\|\leq\| p \|_{\alpha}$, where we have made use of the fact that $\left\|T^{k}\right\|=1 / k$ ! for all $k \in \mathbf{N}_{0}$. Since the polynomials are dense in $A_{\alpha}$, we obtain the desired functional calculus for $T$ on $A_{\alpha}$ by extending $\Phi$ continuously to $A_{\alpha}$.

In view of the preceding counterexample, it seems natural to restrict the attention to functional calculi on algebras of functions which contain at least all the $\mathscr{C}^{\infty}$-functions. Hence, let us consider now a natural Banach function algebra $A$ on some compact subset $K$ of $\mathbf{C}$ and let us assume that, for some integer $k \geq 0$, all the restrictions to $K$ of the $\mathscr{C}^{k}$-functions on $\mathbf{C}$ are contained in $A$. Then $A$ is certainly semi-simple and regular. It therefore follows from Theorem 2.3 of [13] that for every continuous unital homomorphism $\Phi: A \rightarrow \mathscr{L}(X)$ and for every $g \in A$ the operator $T=\Phi(g) \in \mathscr{L}(X)$ is super-decomposable. It is natural to ask whether the representation (12) of the spectral maximal spaces remains valid in this more general setting. Of course, one may try to extend the proof of 3.1 from the special case $g=Z$ to the case of an arbitrary $g \in A$, but this method doesn't work for some of the examples we have in mind. Another possible approach is based on the idea of composition. Assume that $g \in A$ has the property that for each $f \in \mathscr{C}^{\infty}(\mathbf{C})$ the composition $f \circ g$ belongs to $A$ and that the corresponding mapping from $\mathscr{C}^{\infty}(\mathbf{C})$ into $A$ is continuous. Then it makes sense to consider the mapping $\tilde{\Phi}: \mathscr{C}^{\infty}(\mathbf{C}) \rightarrow \mathscr{L}(X)$ given by

$$
\tilde{\Phi}(f)=\Phi(f \circ g) \text { for all } f \in \mathscr{C}^{\infty}(\mathbf{C}) \text {, }
$$

which is certainly a continuous homomorphism satisfying $\tilde{\Phi}(Z)=$ $\Phi(g)$. Hence, under the given assumptions, the operator $T=\Phi(g)$ is generalized scalar so that the algebraic representation (12) of its spectral maximal spaces holds by Theorem 3.1. It can be checked that this method works for many interesting Banach function algebras $A$ including, for instance, the algebras $\mathscr{C}^{k}(I), \mathscr{C}^{k, \alpha}(I)$, and $A C(I)$. However, in the case of the Banach algebra $A(T)$ of all continuous functions on the unit circle $\mathbf{T}$ having an absolutely convergent Fourier series, the indicated method fails, since it is known [11] that only the realanalytic functions operate on $A(\mathbf{T})$. Whether in $A(\mathbf{T})$ multiplication by $g \in A(\mathbf{T})$ can have a divisible subspace appears to be open. A solution to the following problem would be quite interesting.

3.6. Problem. Let $\Phi: A(\mathbf{T}) \rightarrow \mathscr{L}(X)$ be a continuous homomorphism such that $\Phi(1)=I$, and consider the operator $T=\Phi(g) \epsilon$ $\mathscr{L}(X)$ for an arbitrarily given $g \in A(\mathbf{T})$. Does it follow that the 
representation (12) holds for some integer $p$ or at least that $T$ has no divisible subspace different from zero?

We turn finally to the case of spectral operators in the sense of Dunford; see [6] for the basic theory of these operators. The following result was obtained by Foias and Vasilescu in [8] under certain restrictions on the underlying Banach space or on the Boolean algebra of projections corresponding to the spectral measure. These were necessary since their approach was based on Badé's multiplicity theory [6]. Here the exponents are optimal by Example 2.10 of [8].

3.7. TheOREM. If $T \in \mathscr{L}(X)$ is scalar, then

$$
X_{T}(F)=\bigcap_{\lambda \in \mathbf{C} \backslash F}(T-\lambda)^{2} X \text { for all closed } F \subseteq \mathbf{C} .
$$

Moreover, if $T \in \mathscr{L}(X)$ is spectral of type $m \in \mathbf{N}_{0}$, then

$$
X_{T}(F)=\bigcap_{\lambda \in \mathbf{C} \backslash F}(T-\lambda)^{m+2} X \text { for all closed } F \subseteq \mathbf{C} .
$$

Proof. We shall combine certain ideas from the proof of Theorem 3.1 and from [17]. First observe that spectral operators are superdecomposable by Proposition 2.1 of [13]. Hence it follows from Proposition 1.1 that it suffices to prove that

$$
\bigcap_{\lambda \in \mathbf{C}}(T-\lambda)^{2} X=\{0\}
$$

for an arbitrary scalar operator $T \in \mathscr{L}(X)$. Let $E$ denote the corresponding spectral measure, fix an arbitrary

$$
y \in \bigcap_{\lambda \in \mathbf{C}}(T-\lambda)^{2} X
$$

and take some continuous linear functional $\varphi$ on $X$. By the HahnBanach theorem, it suffices to show that $\varphi(y)=0$. We follow the lines of the proof of Theorem 3.1 to obtain a decreasing sequence of squares $P_{n} \subseteq \mathrm{C}$ of side length $s\left(P_{n}\right)=2^{-n} s\left(P_{0}\right)$ such that

$$
|\varphi(y)| \leq 4^{n}\left|\varphi\left(E\left(P_{n}\right)\right) y\right| \text { for all } n \in \mathrm{N} \text {. }
$$

Let $\lambda \in \mathbf{C}$ be the intersection of all the squares $P_{n}$, and choose some $x \in X$ such that $y=(T-\lambda)^{2} x$. Let $\mu$ denote the variation of the complex measure $\varphi(E(\cdot) x)$. Then it follows that

$$
|\varphi(y)| \leq 4^{n} \int_{P_{n}}|Z-\lambda|^{2} d \mu=4^{n} \int_{P_{n} \backslash\{\lambda\}}|Z-\lambda|^{2} d \mu
$$


for all $n \in \mathbf{N}$. Now an obvious application of the Lebesgue dominated convergence theorem shows that the right hand side of this inequality tends to zero as $n \rightarrow \infty$. We conclude that $\varphi(y)=0$, which completes the proof.

Let us note finally that for spectral operators on Hilbert spaces the exponents of the preceding result can be improved due to the rather special geometry of Hilbert spaces; for details we refer to [3] and [17].

\section{REFERENCES}

[1] E. Albrecht, J. Eschmeier, and M. M. Neumann, Some topics in the theory of decomposable operators, Operator Theory: Advances and Applications, 17; Birkhäuser, Basel (1986), 15-34.

[2] E. Albrecht and M. M. Neumann, Continuity properties of $C^{k}$-homomorphisms, Radical Banach algebras and automatic continuity; Springer Lect. Notes in Math. 975 (1983), 356-374.

[3] E. Albrecht and F.-H. Vasilescu, Non-analytic local spectral properties in several variables, Czech. Math. J., 24 (1974), 430-443.

[4] I. Colojoară and C. Foiaş, Theory of Generalized Spectral Operators, Gordon and Breach, New York 1968.

[5] H. G. Dales and A. M. Davie, Quasianalytic Banach function algebras, J. Funct. Anal., 13 (1973), 28-50.

[6] N. Dunford and J. T. Schwartz, Linear Operators, Part III: Spectral operators, Wiley-Interscience, New York 1971.

[7] I. Erdelyi and Wang Shengwang, A local spectral theory for closed operators, London Math. Soc. Lect. Notes Series 105, Cambridge University Press, Cambridge 1985.

[8] C. Foiaş and F.-H. Vasilescu, Non-analytic local functional calculus, Czech. Math. J., 24 (1974), 270-283.

[9] B. E. Johnson, Continuity of linear operators commuting with continuous linear operators, Trans. Amer. Math. Soc., 128 (1967), 88-102.

[10] - Continuity of homomorphisms of Banach G-modules, Pacific J. Math., 120 (1985), 111-121.

[11] Y. Katznelson, An Introduction to Harmonic Analysis, Second edition, Dover, New York 1976.

[12] K. B. Laursen, Algebraic spectral subspaces and automatic continuity, to appear in: Czech. Math. J., (1987/88).

[13] K. B. Laursen and M. M. Neumann, Decomposable operators and automatic continuity, J. Operator Theory, 15 (1986), 33-51.

[14] B. Malgrange, Ideals of Differentiable Functions, Oxford University Press, London 1966.

[15] M. M. Neumann, Decomposable operators and generalized intertwining linear transformations, to appear in: Operator Theory: Advances and Applications; $(1987 / 88)$.

[16] A. G. O'Farrell, Polynomial approximation of smooth functions, J. London Math. Soc., (2) 28 (1983), 496-506. 
[17] V. Pták and P. Vrbová, On the spectral function of a normal operator, Czech. Math. J., 23 (1973), 615-616.

[18] M. Putinar, Hyponormal operators are subscalar, J. Operator Theory, 12 (1984), 385-395.

[19] C. R. Putnam, Ranges of normal and subnormal operators, Michigan Math. J., 18 (1971), 33-36.

[20] W. Rudin, Real and Complex Analysis, McGraw-Hill, New York, 1966.

[21] A. M. Sinclair, Automatic continuity of linear operators, London Math. Soc. Lect. Notes Series 21, Cambridge University Press, Cambridge 1976.

[22] F.-H. Vasilescu, Analytic Functional Calculus and Spectral Decompositions, D. Reidel Publ. Comp., Dordrecht and Editura Academiei, Bucureşti 1982.

[23] P. Vrbová, Structure of maximal spectral spaces of generalized scalar operators, Czech. Math. J., 23 (1973), 493-496.

Received January 22, 1988. Research supported by the U.S. National Science Foundation (NSF) under Grant No. DMS 86-03001 and by the Deutsche Forschungsgemeinschaft (DFG) under Grant No. Ne 249/2-2.

UNIVERSITY OF CALIFORNIA

LOS ANGELES, CA 90024

U.S.A.

AND

Southwest Missouri State University

SPRINGFIELD, MO 65804

U.S.A. 



\section{PACIFIC JOURNAL OF MATHEMATICS EDITORS}

\author{
V. S. VARADARAJAN \\ (Managing Editor) \\ University of California \\ Los Angeles, CA 90024 \\ Herbert Clemens \\ University of Utah \\ Salt Lake City, UT 84112 \\ THOMAS ENRIGHT \\ University of California, San Diego \\ La Jolla, CA 92093
}

R. FINN

Stanford University

Stanford, CA 94305

HeRmanN FlaschKa

University of Arizona

Tucson, AZ 85721

VAUGHAN F. R. JONES

University of California

Berkeley, CA 94720

STEVEN KeRCKHOFF

Stanford University

Stanford, CA 94305
RoBION KIRBY

University of California

Berkeley, CA 94720

C. C. MOore

University of California

Berkeley, CA 94720

HAROLD STARK

University of California, San La Jolla, CA 92093

\section{ASSOCIATE EDITORS}
R. ARENS
E. F. BECKENBACH
B. H. NeUmanN
F. WolF
K. YosHIDA (1906-1982)

\section{SUPPORTING INSTITUTIONS}
UNIVERSITY OF ARIZONA
UNIVERSITY OF BRITISH COLUMBIA CALIFORNIA INSTITUTE OF TECHNOLOGY UNIVERSITY OF CALIFORNIA MONTANA STATE UNIVERSITY UNIVERSITY OF NEVADA, RENO NEW MEXICO STATE UNIVERSITY OREGON STATE UNIVERSITY
UNIVERSITY OF OREGON UNIVERSITY OF SOUTHERN CALIFORNIA STANFORD UNIVERSITY UNIVERSITY OF HAWAII UNIVERSITY OF TOKYO UNIVERSITY OF UTAH WASHINGTON STATE UNIVERSITY UNIVERSITY OF WASHINGTON 


\section{Pacific Journal of Mathematics}

\section{Vol. 137, No. $1 \quad$ January, 1989}

V. S. Varadarajan, Henry Abel Dye $\ldots \ldots \ldots \ldots \ldots \ldots \ldots \ldots \ldots \ldots$ iii

Huzihiro Araki, An application of Dye's theorem on projection lattices to orthogonally decomposable isomorphisms $\ldots \ldots \ldots \ldots \ldots \ldots \ldots \ldots \ldots$

Richard Arens, The limit of a sequence of squares in an algebra need not be

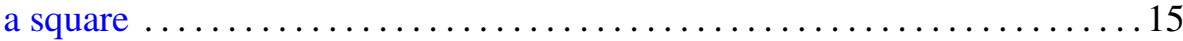

William Arveson, An addition formula for the index of semigroups of

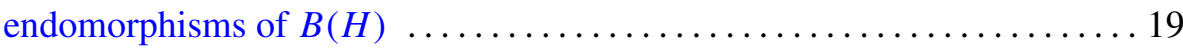

Robert James Blattner and Susan Montgomery, Crossed products and

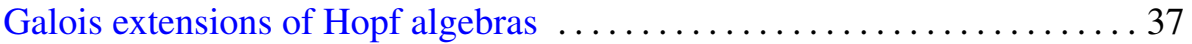

Erik Christensen and Allan M. Sinclair, On the vanishing of $H^{n}\left(\mathscr{A}, \mathscr{A}^{*}\right)$

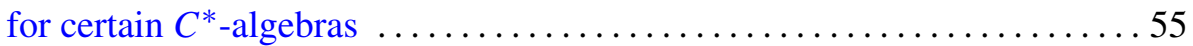

Philip C. Curtis, Jr. and Michael M. Neumann, Nonanalytic functional

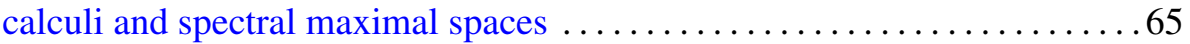

George A. Elliott and David E. Handelman, Addition of $C^{*}$-algebra extensions

Yaakov Friedman and Bernard Russo, Some affine geometric aspects of

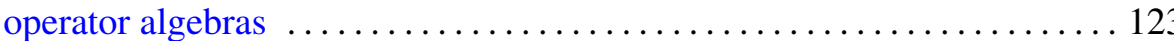

Valentin Ya. Golodets and Sergey D. Sinelshchikov, Regularization of actions of groups and groupoids on measured equivalence relations . . . 145

Irving Kaplansky, CCR-rings 155

Hideki Kosaki, Characterization of crossed product (properly infinite case)

Gert Kjærgaard Pedersen, Three quavers on unitary elements in $C^{*}$-algebras

Sorin Popa, Relative dimension, towers of projections and commuting squares of subfactors

Martin E. Walter, On a new method for defining the norm of

Fourier-Stieltjes algebras 